\title{
Psychosocial Skills in a Youth Soccer Academy: A Holistic Ecological Perspective
}

\author{
Carsten Hvid LARSEN ${ }^{1} \bullet$ Dorothee ALFERMANN ${ }^{2}$ \\ Mette Krogh CHRISTENSEN ${ }^{1}$
}

\begin{abstract}
bjectives: The latest research in talent development show that more varied psychological and especially social and cultural circumstances play an important role in talent development. This study assumes an ecological approach to explore which psychosocial skills are important in a youth soccer academy and how psychosocial skills are practiced in this particular environment. Method: The research takes the form of a case study. Data were collected from multiple perspectives (in-depth interviews with managers, coaches and players), from multiple situations (observation of training, competitions and meetings) and from the analysis of documents. Results: The findings reveal explicit (being practiced and talked about) and implicit (indirectly practiced and talked about) psychosocial skills in the youth soccer academy and a differentiation between internal and interpersonal psychosocial skills. Conclusions: Important explicit psychosocial skills are motivation, self awareness and the ability to work hard. However, even more important for the young soccer players dealing with the transition to professional soccer seem to be implicit psychosocial skills such as managing performance and process outcomes (internal) and the ability to utilize team skills and general social skills (interpersonal). Despite the fact that the environment expects the young players to display these skills, they are only indirectly practiced. This study demonstrates the interweaving of psychosocial skills and practice in the talent development environment, and thus underlines the social construction of psychosocial skills in elite sport.
\end{abstract}

Keywords: soccer, athletic talent development environment, career transition, organizational culture, talent development, sport psychology and leisure.

1 Institute of Sport Science and Clinical Biomechanics, University of Southern Denmark, Odense, Denmark

2 Faculty of Sport Science, Leipzig University, Leipzig, Germany 


\section{Psychosocial skills in a youth soccer academy: a holistic ecological perspective}

The latest research in talent development has shown that more varied psychological and especially social and cultural circumstances play an important role in talent development (Côté, Lidor, \& Hackfort, 2009). The achievement of excellent performance is about handling the diversity of challenges during everyday life (Holt \& Dunn, 2004). Adolescence is the biographical period of life in which investment in great performances should be achieved and this phase of development is especially demanding for the athlete. The young athletes who take responsibility for their own careers also experience a great amount of pressure in school during this period (Brettschneider, 1999) and are expected to balance what are often contradictory demands in sport and school (Christensen \& Soerensen, 2009). One only has to look at the number of successful juniors who have failed to make the transition to senior sport and have either dropped out of the sport or compete at lower levels (MacNamara, 2011). The path to professional sport is complex and demanding for players of youth soccer (i.e. football) and only $20 \%$ of teenage players recruited to professional teams are still in these teams beyond their teen years (MacNamara, 2011). The athlete's resources and psychological skills are a key factor for coping in future transitions (Alfermann \& Stambulova, 2007). In addition to athletic skills, psychosocial skills are key determinants of those who emerged as talented athletes, maintained excellence and were able to balance sport and school in the process (Henriksen, Stambulova, \& Roessler, 2010). Research of training programs highlight that it is necessary to incorporate a holistic "skills package" containing a wide variety of cognitive, perceptual and motor skills (Janelle \& Hillman, 2003), as these "teachable" factors are important in distinguishing the best performers at later stages (Helsen, Hodges, Winckel, \& Starkes, 2000). However, in order to establish effective youth programs and match transition demands there is a need to know which psychosocial skills are needed by adolescent soccer players.

Reviewing relevant literature, psychosocial skills are often associated with terms such as life skills, positive youth development, social-emotional growth (Gould \& Carson, 2008), psychological skills (Danish et al., 1995, p. 23), psychological characteristics (MacNamara, Button \& Collins, 2010; MacNamara, 2011), psychosocial competencies and assets (Harwood, 2008). Despite this lack of a precise definition psychosocial skills are, in this case, a key determinant in the talent development process (MacNamara et al., 2010) and are competencies divided into internal and external assets. According to Harwood (2008), internal assets represent attributes of the individual such as commitment to learning, goal setting, emotional control, self-esteem, hard work ethic or interpersonal competence (e.g., communication skills or social skills), whereas external assets 
are characterized by the quality of the environment shaping the child, including access to positive role models, social support, and positive peer influence. In addition, an early emphasis upon psychosocial skills in soccer may enhance the "young player" alongside equipping the "young person" with internal skills transferable to other life domains (Harwood, 2008).

An ecological approach to exploring psychosocial skills. This study assumes an ecological approach (Bronfenbrenner, 1999) to explore which psychosocial skills are important and practiced in the environment of a youth soccer academy. From an ecological perspective it makes no sense to perceive psychosocial skills or coping strategies as inner, independent and stable personalities. Instead, psychosocial skills are perceived as socially constructed, culturally contingent, and highly dependent on the specific environmental conditions. An ecological perspective shifts the perspective away from the individual athlete to the context in which the athlete is developing. Bronfenbrenner's ecological systems theory represents an important cornerstone of modern developmental science (Bronfenbrenner, 1979). It is a basic premise of ecological systems theory that development is a function of forces emanating from multiple settings and from relations between these settings (Bronfenbrenner, 1999). The theory is based on four main concepts: Person, process, context, and time, and it regards the dynamic relationships in the person-process-context-time interconnection. Person-process-context-time is based on individual characteristics (e.g. psychosocial skills), proximal process (e.g. activities and practice in the environment), contextual variables (e.g. the environment in which the activities are happening), and the temporally evolving nature of relations between talented athletes and different levels of their environment. The talented athletes interact in proximal micro systems (e.g. patterned activities, roles and interpersonal relations that a person experiences in a setting), meso systems (e.g. interrelations among major settings such as training and matches in the soccer setting and lessons in the school setting), exo systems (e.g. other specific formal and informal social structures) to distal macro systems (e.g. overarching institutional patterns of the culture or subculture) (Bronfenbrenner, 1979; Bronfenbrenner, 1999). This article aims to uncover the development of psychosocial skills in young soccer players in relation to their micro-, meso- and macro environment, and intends to explore (a) which psychosocial skills are important in a youth soccer academy and (b) how these psychosocial skills are practiced in the environment.

\section{Method}

The study was organized as an explorative integrative and qualitativelyoriented case study in a successful Danish soccer environment. A case study allows researchers to retain the holistic and meaningful characteristics of real- 
life events (Yin, 2009). Moreover, Kruuse (2008) argues that case studies are meaningful if complex issues are to be investigated in which many different factors can influence the results and where it is not possible to control these variables. For these reasons, the case study was an appropriate methodological choice for this study.

\section{The case study}

Our overall approach to the case study is the so called explorative integration, which is a cyclic approach of a continuous dialogue between prechosen theories, generated data, our interpretation and feedback from our informants, which will hopefully lead to more inclusive theory building or even understanding (Maaloe, 2004). The case study is a strategy to empirically explore chosen contemporary phenomena in its natural context by using sources of data that can be used as proof of evidence (Robson, 2002). Maaloe (2004) defined a case study as an "empirical inquiry that investigates a real-life phenomenon within its real-life context" (p. 5). Furthermore, researching into "the natural occurring experiments" (Yin, 2009, p. 4), means studying phenomena that we do not control while we study them. According to Maaloe (2004), case studies are focused on three main types of phenomena: Systems, courses of action and persons, and therefore case studies are the only way to explore links between events, reactions (including decisions, emotions and reflections) and behaviors as they emerge in real-life situations. Accordingly, this case study used different sources of data (observations of actions, interviews with different persons and analysis of documents in the environment) and via triangulation we applied several analysis methods of the multifaceted data about the same phenomenon. Furthermore, the multiple source strategy provided us with the means to distinguish between private and shared perceptions of what was going on within the environment as well as evidence of what it means to those involved.

\section{Case selection}

On the basis of expectations about its information content (Flyvbjerg, 2006), we selected one of the oldest and most successful Danish soccer clubs (AGF) and its affiliated players, staff, and nearby environment in the city of Aarhus for the case study. We used an information-oriented case selection method known as "paradigmatic case selection", that is the case is selected with the aim "to maximize the utility of information from small samples and single case [and] to develop a metaphor or establish a school for the domain that the case concerns" (Flyvbjerg, 2006, p. 230). Aarhus is the second largest city in Denmark, and it has one of the highest concentrations of talented and elite athletes in Denmark. The club is part of ESAA (Elite Sport Academy Aarhus), 
which is an institutional set-up that provides young talented athletes with the opportunity to combine an educational career with an elite sports career. ESAA is based on a partnership model with the local elite sport clubs, local educational institutions and the national sport federations. In 2010-2011 ESAA co-operates with 15 different sports and more than 350 athletes, 2 primary schools, 9 secondary schools (gymnasium and vocational) and the University of Aarhus (ESAA, 2011) ${ }^{1}$.

\section{Procedure}

Contact was made with the sports director of ESAA, and in corporation with him the club was selected. After selection of the club, preliminary acceptance was gained through the manager of sports and coaches. The identity of the club is known but the identities of youth players and other persons involved in the club are anonymous ${ }^{2}$.

\section{Description of the case}

The club consists of two departments: A volunteer, non-elite department for a wide range of soccer players, and a professional elite department for male youth teams ranging from under-13 to under-19, plus senior teams. The club has fulltime coaches for each youth team. The professional senior team is organized into a section of its own. The professional senior team is playing in the Danish Premier League and a reserved team in a separate tournament for professional teams in Denmark. In the professional senior team there is a fulltime first team coach, assistant coach and relevant experts supporting the team and staff. A team of coaches dictates the strategy for talent development and new perspectives for the youth teams. The old club house shows the stories and traditions of the club. The club is a self-contained part of the largest sports club in Aarhus (Aarhus Gymnastics Association) which was founded in 1880 as a multidiscipline sports club. Soccer joined the program in 1902. The club is one of the most successful Danish soccer clubs with 5 Danish championships and 9 Cup titles (a record). The professional department of AGF was founded in 1978. In 2005, the professional soccer department joined the newly consolidated Aarhus Elite and expanded the business to other areas; Aarhus GF (handball), Bakken Bears (basketball) and Atletion (Stadium and office buildings). AGF has a successful record for producing male youth national players and is successful in helping talented youth players to make the transition to professional soccer. This study focused on the under-17 team and its staff in the professional department. 


\section{Data Collection}

Data was collected from multiple perspectives. Firstly, participant observation (Spradley, 1980) was used as the primary data collection method in the case study. The observations were performed by the principal researcher as a "moderate participant" (Spradley 1980, p. 60). We chose him to be the moderate participant because he is an educated PE teacher and a trained soccer player. We assumed that these qualities would enhance the possibilities of being a successful moderate participant in the chosen environment. As a moderate participant, he observed day-to-day training, acted as an assistant coach and sparring partner for the under-17 coach for 21 days during 7 months. The primary researcher had to be aware of the social reality and the perception of the reality by the participants (Olesen, 2001). In the observations the principal researcher focused on observing deeper structures and forces that create social displacements. Interpretations of actions, relations between the parts of the environment and the interests in the environment were main foci. Therefore the observations were based on real time and in real life contexts. The challenge was to be aware of and confront the straightforward perceptions and the apparent perceptions as they were shown in everyday actions in the environment. During the observations the principal researcher used descriptive field notes to capture different perspectives and variation in the information of the participants (Patton, 2002).

Secondly, the primary researcher conducted 15 individual interviews, which lasted between 40 and 90 minutes. Interviewees were selected on the basis of interviewing in both the micro- and macro environment. Most of the interviewees are connected to the club, which represents the closest and most important relation to youth players. Furthermore, school and sport coordinators from primary and upper secondary schools were interviewed for highlighting the communication and relation between school and club. The interviews were semi-structured (Kvale \& Brinkmann, 2009) which allowed the interviewees freedom to discuss issues that were personally important to them. Within the semi-structured format open-ended questions were used to yield in-depth responses about the interviewees' experiences, perceptions and knowledge about the environment and psychosocial skills. Using the semi-structured approach, the principal researcher had questions on a schedule, but was guided by the schedule rather than dictated by it (Patton, 2002).

Finally, archives and documents were used as a substantial category of data in the case study (Ramian, 2007). The documents included were newspaper articles involving talent development in the club, homepage of the club, training plans, season plans, calendar, internal information about talent development, match evaluations, match statistics, documents from the municipality involving 
sport and school, yearly reports from the municipality and the club's code of conduct. The documents were compared to interviews and observations in order to establish evidence for important psychosocial skills in the environment (see Table 1, for a full review of the collected data and participants).

Table 1. Collected data in the survey

1 manager of sports; 1 club manager; 1 professional coach; 2 youth coaches; 4 youth players; 2 profession-

Interviewees al players; 1 school and sport coordinator in primary school; 2 school and sport coordinators in upper secondary school and 1 consultant from the municipality.

Observation time

170 hours from main season to off season and from pre-season to peak season.

Activities

Practice; competition; training camp; practicing alternative sports in school; during school sessions; and youth player education seminar.

Informal talks during observations

Youth players, professional players, coaches, managers, administrators, parents, club staff and school coordinators

Club mission statements, articles involving talent development, homepage of the club, training plans, season plans, calendar, internal information about tal-

Archival data used ent development, match evaluations, match statistics, documents from the municipality involving sport and school, yearly reports from the municipality and the clubs code of conduct.

\section{Data analysis}

The interviews were audio recorded and transcribed verbatim. Transcriptions were subsequently sent to the participants for verification. Full anonymity was guaranteed to the participants in the study. The data analysis were based on an abductive strategy (Dubios \& Gadde 2002; Chamberlain, 2006) and consisted of three steps using Nvivo 8 coding software. The first step consisted of a categorization (Kvale \& Brinkmann, 2009, p. 201) of psychosocial skills in either internal or interpersonal, inspired by Harwood (2008). The second step was a theoretical reading (Kvale \& Brinkmann, 2009, p. 238) of data in the two 
categories with the purpose of generating new low order themes. This step was inspired by Jones and Lavallee (2009) and generated dimensions of familiar phenomena as well as new low order themes (Kvale \& Brinkmann, 2009, p. 238). The first two steps of the analysis were based on data from the interviews. The third step consisted of a more open reading of data and revealed a deeper layer consisting of explicit (being practiced and talked about) and implicit (indirectly practiced and talked about) psychosocial skills. This step of the analysis was mostly based on observations, archives and documents, and it established ecological validity to the data (Bronfenbrenner, 1979).

An on-going member-checking process with coaches was also completed. The coaches and manager received a full, written copy of the anonymous results, which they discussed with the principal researcher. This member-checking process (Lincoln \& Guba, 1985) as well as peer validity (Kvale \& Brinkmann, 2009), with the second and third author helped establish the accuracy of our interpretations. The triangulation of data sources (i.e., coach's perspectives) and data collection techniques (i.e., observation and interview) helped to establish the trustworthiness of the analysis and findings (Patton, 2002).

\section{Results}

Our analysis of data resulted in an overall distinction between explicit and implicit psychosocial skills across two high order themes: internal and interpersonal (Harwood, 2008). The interview text revealed that participants spontaneously and to a large degree express and talk about internal psychosocial skills, while reports on interpersonal psychosocial skills were often initiated by the principal researcher's questions from the interview guide. This may explain the amount of interview text skewed towards internal low order themes (especially self awareness and ability to work hard). However, this does not mean that we can conclude internal psychosocial skills are more important than interpersonal psychosocial skills (see table 2), only that the participants related straightforwardly more aspects of internal psychosocial skills rather than interpersonal psychosocial skills as a part of the shared articulated discourse in this particular environment. Furthermore, our observations and the informal talks during observations revealed that social skills in particular seemed to be more or less implicit in the environment. Consequently, we present the results in two high order themes: internal and interpersonal psychosocial skills. The descriptions of the appertaining low order themes include analysis of explicit and implicit aspects of each low order theme. Table 2 shows a matrix of our interpretation and categorization of data into the two main pairs of themes: internal and interpersonal psychosocial skills as well as explicit and implicit psychosocial skills, and the appertaining low order themes. 
Sport Science Review, vol. XXI, No. 3-4, August 2012

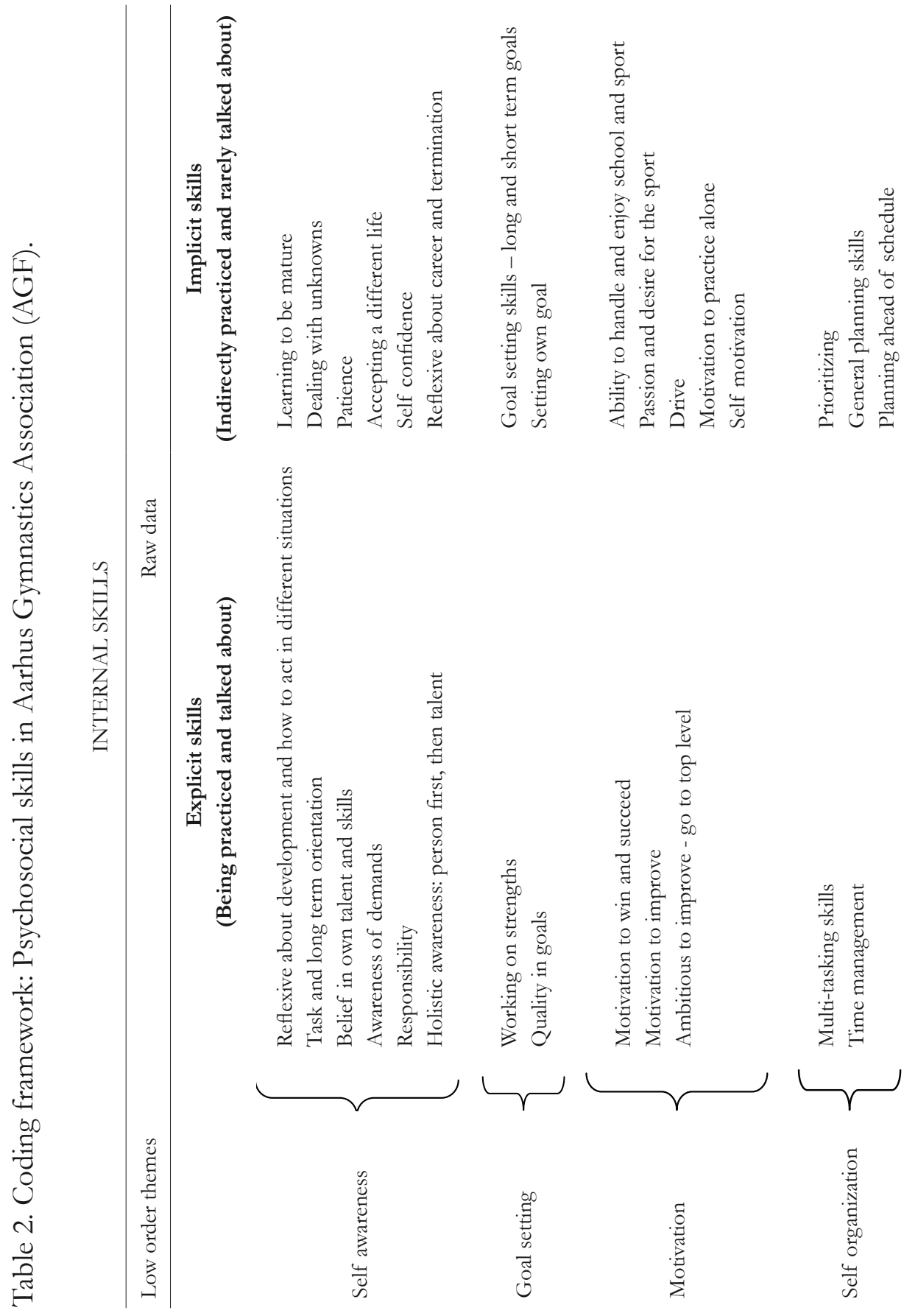


Psychosocial Skills in Youth Soccer

อี
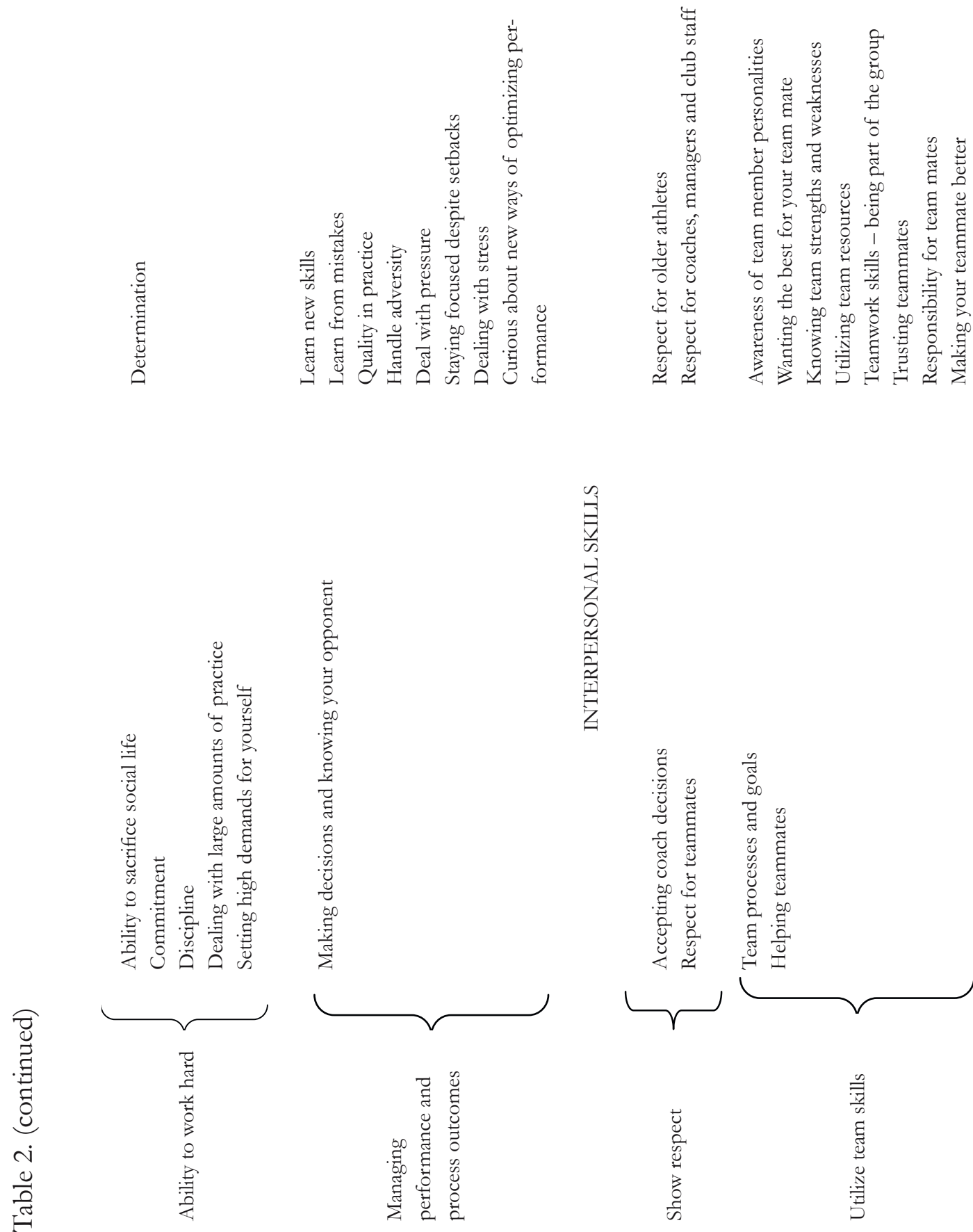
Sport Science Review, vol. XXI, No. 3-4, August 2012

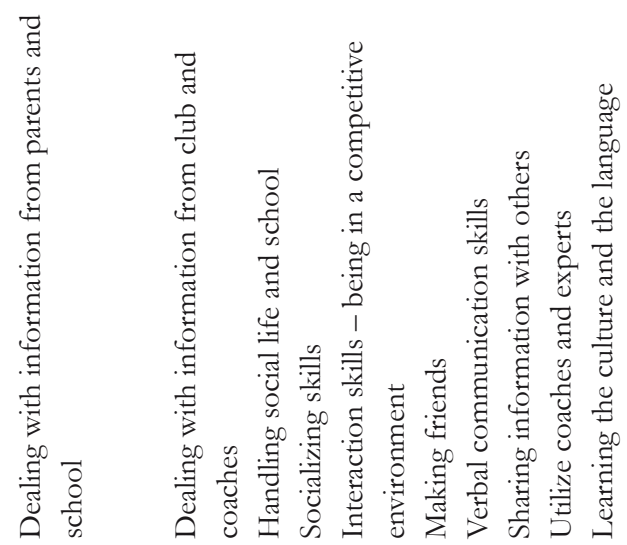

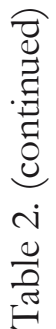

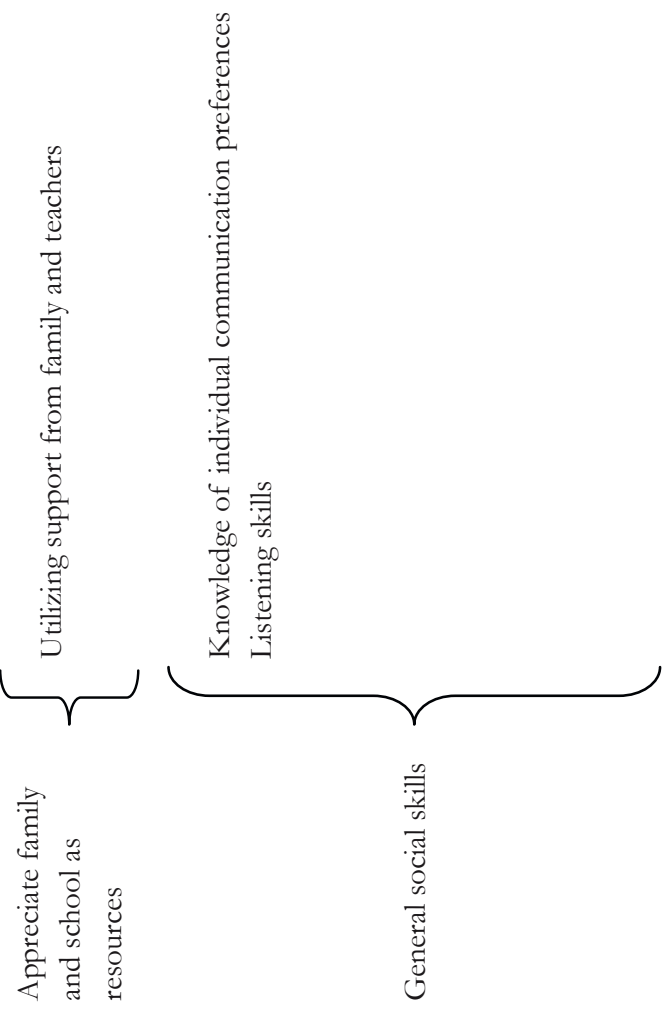


In the following, words marked with quotation marks are raw data (the participants' own words) from table 2.

\section{Internal psychosocial skills}

Within the internal category we generated six low order themes and these include self awareness, goal setting, motivation, self organization, ability to work hard and managing performance and process outcomes.

Self awareness. All participants talked about the importance of self awareness during practice and as a talented soccer player in general. The manager and the coaches said they select and develop players that are aware of their competencies on different levels (e.g. different situations and domains, short and long term development, demands in the environment) and know how to develop them. One of the youth coaches also emphasized that a talented player needs to be "aware of the demands" of the sport and what is needed to "survive" in a professional club. Another youth coach described self awareness as the ambition and eagerness to be better and be aware of what the player himself wants to improve when he is on the pitch as well as off the pitch:

Well, it is the ambition to improve and practice. We use an expression that there is a difference if a player goes to practice and shows an understanding of why they are doing what they are doing instead of just being coached. There is a difference between the outcome and quality of the practice and the development of the player. If the player is able to coach themselves, they will improve on and off the pitch (Youth coach).

The overriding and explicit discourse among the coaches is about the players' need for self awareness in order to survive, that is, youth players have to "believe in their own talent and skills" and be "reflexive about development" and "how to act in different situations". Additionally, the coaches emphasized the necessity that both the individual player and the systems surrounding the player consider a "holistic awareness" in their ongoing development of soccer skills. Our observations support this idea that the holistic perspective is vital and a dominant endeavor in the soccer environment and also in the relation between the schools affiliated with ESAA and the club. The primary school coordinator explained that the club and the schools have a tradition of easing the everyday life for the players, because the school's values and norms are in line with those of the club. Self awareness is an important psychosocial skill on the path to success in this environment and is constructed on a holistic philosophy expressed in documents and yearly reports from the municipality. The philosophy represents a coherent and strong focus on individuality as 
well as responsible career- and talent development with accentuation of the development of the individual player's self awareness and ability to be reflexive. Both the club and the school seem to echo this philosophy - at least it is present in the explicit discourse among the participants in this case study. A consultant in the municipality expressed the importance of creating awareness in the entire environment (coaches, teachers, coordinators, family) that talent development and the path to professional soccer is centered around the youth soccer player as a whole person: "Person first, then talent [...] school and sport are equally important" (Consultant from the municipality). He continued about a need of a holistic approach in the environment in general:

It is about providing the young athletes with the fundamental knowledge about the complexity and wholeness of things. If a young athlete removes a part of the environment then he has to know that something is missing in the whole (Consultant from the municipality).

During our observations and in the informal talks we gained deeper understanding of implicit aspects of self awareness as a psychosocial skill. The players are "dealing with unknowns" about their future on the first team, they have to display "patience" and "self confidence" in order to maintain their position in the club, and they have to "learn to accept a different life" than their peers. In short, they have to "learn to be mature" while pursuing the boyish dream of being a professional soccer player.

Goal setting. Youth players, coaches and elite players all expressed the importance of goal setting as an important skill in the transition from talent to professional player, and according to the club manager and coaches the primary objective for the club is to "work on strengths" and ensure "quality in goals". The professional coach mentioned the importance of modern soccer players to be able to set goals: "Soccer is like climbing a mountain. You have to climb up all the time. On your way up there is hardship and adversity and all the time you have to set new goals" (Professional coach). One of the professional players shared his perspective and agreed that youth players need to have general goal setting skills: "If you set goals for yourself, you kind of put down on paper, exactly what you want to do, and you can see it in front of you [...] your mind kind of sets to it (ed. improving)" (Professional player). Even if the participants constantly report the importance of goal setting skills, we did not observe actual teaching or practicing of goal setting during the training or in other areas of practice. This is an example of how the environment is able to talk about goal setting as a psychosocial skill without teaching or practicing it explicitly. In this way, the explicit psychosocial skill of goal setting seems to function as an guideline something to talk about because it underpins the self understanding in the club, 
but the players mostly are left alone with "setting their own goal" and thus the actual goal setting skill becomes an implicit psychosocial skill.

Motivation. The participants mention motivation as a key quality in a youth player. One of the older professional players related:

I think ambition is something that's really important. I think you need the motivation, want to achieve something and get better and get to the top level. I think if you don't have that real ambition to get somewhere; it is going to be tough to get there (ed. top level soccer) [...] so I think it's really important to have ambition and motivation to succeed and get to another level, and continue to improve yourself (Professional player).

Some participants describe the psychosocial skill of motivation in terms of "ambition to improve and go to the top level" and "motivation to improve" every day. It is clear from our data that the youth players know that they need to improve. However, some aspects of motivation as a psychosocial skill (e.g. the passion and desire for the sport, motivation to practice alone) were only indirectly practiced and talked about in very informal off pitch talks between players and coaches, and we did not observe situations in training or in coaching dialogues where the primary focus was the players' learning of motivation as a psychosocial skill beyond showing eagerness to improve one's own soccer skills in the actual training sessions or in matches.

Self organization. When referring to self organization it is deemed crucial, coaches, school coordinators and the municipality underlined again and again, that youth players are able to structure everyday activities and are able to multitask. As an upper-secondary school coordinator recounted: "They have to be able to use a calendar [...] arrange the day and make it work, [...] plan and be able to manage the pressure and stress in different situations" (Upper-secondary school coordinator). Another coordinator from upper-secondary school agreed that the players have a stressful day and it is a challenge to manage school and sport: "Besides school they practice about 15-30 hours, so they have to be very good at organizing the day" (Upper-secondary school coordinator). A youth player expressed the relation between the ability of structuring your day and what is needed to do it successfully, "It demands structure during the day. It demands concentration throughout the day [...] you need proper nutrition in order to have the energy to handle school and soccer one hundred percent" (Youth player). Despite the clearly expressed need for self organization we did not observe direct teaching or guidance with regards to self organization as a psychosocial skill (e.g. prioritizing, general planning skills, and the ability to plan ahead of schedule). However, the players' multitasking skills and time management skills 
were often talked about and addressed on a regular basis in school, before training sessions, at the ESAA information meeting, and by coaches in the club, but the frequent mentioning of the need for self organization seemed to be an intangible reminder rather than a tangible support to the players.

Ability to work hard. The participants agreed that the ability to work hard is a fundamental skill to be able to successfully coordinate and excel in school and soccer. An upper-secondary coordinator acknowledges that the youth players handle dual careers (school and sport) and that demands a certain mentality. The club philosophy is directed towards youth players with: "A little less talent but the ability to work very hard" (Manager of sports). It is clear from our data that the ability to work hard is essential for being a successful youth player. During our observations we noted that the players were able to "sacrifice social life", "stay committed", "deal with large amounts of practice" and "set high demands for themselves". Especially the "ability to work hard" was evident in the club before, during, and after training sessions and matches. It is clear from our observations and informal talks that the ability to work hard was a pivotal part of being a successful youth player in the club, and also an integrated part of the identity of an AGF-footballer. To wear the white AGF shirt was synonymous with the ability to work hard.

Managing performance and process outcomes. A youth player described the importance of being able to learn from mistakes and being ambitious about development and process outcomes: "You have to be able to forget mistakes it you want to play at a senior top level [...] You have to set high demands for yourself all the time" (Youth player). Furthermore, the professional coach related what is needed for making the transition to professional soccer:

The players that are curious about new ways to improve are often the ones that want to improve all the time. They ask for advice and have the extra drive, because they want to achieve the extra. And it is often these players that make it (Professional coach).

In general the professional players, club manager and professional coach related that it is crucial to be "able to handle adversity", "learn from mistakes and deal with pressure" in order to make the transition to professional soccer. From our observations there were no direct teachings of these aspects of the psychosocial skill "managing performance and process outcomes", even if this seems an essential psychosocial skill, from the professional players experience, for making the transition to professional soccer. Instead, our observations of the way in which AGF practiced "managing performance and process outcomes" point at only one strategy: The players learn to forget the mistakes and keep up the hard work. 


\section{Interpersonal psychosocial skills}

Within the interpersonal category we generated four low order themes and these include respect, utilizing team skills, appreciating family and teachers as resources, and general social skills.

Show respect. Being a newcomer in the club, the principal researcher was introduced to the history of the club and its preceding legends as well as the almost family-like atmosphere in the club. The early observations and the following dialogues with the participants revealed an underlying value in the club that related to the importance of showing respect for coaches, teammates, older athletes, opponents, referee, officials, personnel and spectators. To show respect for $A G F$ and those engaged in the club seemed to be a pivotal virtue to learn as a member of $\mathrm{AGF}$, including the young players engaged in talent development. One of the professional players expressed the necessity to show respect, but also the importance not to be too respectful when you are a youth player at a professional level: "Show respect, but [...] have confidence in yourself" (Professional player). Another professional player agreed and went on to describe work ethic and responsibility as important skills: "I think the values within the team are showing respect to everybody [...]responsibility [...] people have to be ready to do what they're supposed to do" (Professional player). Even though the ability to show respect for older players is important (at least to the older players themselves), our observations did not show that this psychosocial skill was directly talked about. It was indirectly practiced by the coaches and the older players in their expectations to the younger players - but nobody told the youth players how to show respect and why it was important.

Utilize team skills. Connected to respect was the issue of team skills as an important psychosocial skill for developing and performing in team sport. A youth coach described certain aspects of team skills as team goals and processes involving results, performance and process goals were important skills and were practiced and talked about on a regular basis. Additionally, AGF has a family atmosphere where the focus of the players is to help each other and work towards improvement. The observations showed that it is natural for youth players to help their teammates. One of the prominent young players mentioned that knowledge of individual orientations is important to function on the team. As an ego-oriented player and leader in the team related:

You have to remember to make your teammates better. I am a result-oriented player and when I am communicating with another result-oriented player, as with me, you have to be more specific, maybe using slightly 
harsher language than with a task- or social oriented player, perhaps talking to him during half time (Youth player).

As this youth player relates, "knowledge of team personalities" and other aspects of psychosocial such as "utilizing team skills", "knowing team strengths and weaknesses", "utilizing team resources" and "teamwork skills" were all important but rarely a part of the discourse and explicit teaching during meetings and coaching sessions in the environment. Still these aspects seemed to be important to the youth players' experience of being a part of and performing on the team.

Appreciate family and school as resources. The youth coaches and especially the youth players emphasized the importance of their family and their support networks to be able to balance sport and school. However, our observations did not show extraordinary involvement of the parents compared to what is general to Danish leisure sport. A noteworthy feature in this environment is the coordination and cooperation between sport and school, but also support from teachers at the school. As a youth player said about important supporters:

My teachers are important. My class teacher and sports teacher are very interested in what I do. So they help me a lot. And if there is some classes that I miss, then they don't give me a hard time about it (Youth player).

General social skills. The club manager emphasized the importance of being able to learn from others' experiences, being able to listen and take advice which requires a certain humbleness: "The willingness and openness to learn from others' experiences [...] and to be open to new areas of development" (Manager of sports). A primary school coordinator finds social skills very important because these skills help the youth player build social relations in the different domains that they are part of more easily. Social skills are important to be able seek support and be supported by coaches and teammates.

It is clear from our data that "knowledge of individual communication preferences" and "listening skills", as communicative aspects of general social skills, were practiced and talked about before, during and after practice sessions, games and were also part of the regular discourse in the environment. However, observations established that important aspects such as "being able to handle social life and school", "socializing skills" and "utilizing coaches and experts" were a minor part of the discourse and rarely mentioned by others than the youth players themselves. 


\section{Discussion}

The purpose of this study was to explore which psychosocial skills are important in a youth soccer academy and investigate how psychosocial skills are practiced in the environment. As outlined previously the findings revealed two important results that will be discussed: the categorization of explicit (being practiced and talked about) and implicit (indirectly practiced and rarely talked about) psychosocial skills and the differentiation between internal and interpersonal psychosocial skills.

The results showed a differentiation between explicit and implicit psychosocial skills which were related to the process (the regular activities and practice in the environment) and characteristics of the environment. Research indicates, that it is important that talent development environments know which psychosocial skills are important in academies and whether they are explicitly or implicitly taught (Martindale \& Mortimer, 2011, Henriksen et al., 2010). Schein (1990) pointed out that a culture - in this instance, the academy and soccer environment in AGF - consists of three layers of visibility. In the present study the explicit and practiced psychosocial skills were observable as artifacts and espoused values (norms and ideologies) and were communicated on a frequent basis (Schein, 1990). The implicit and indirectly practiced psychosocial skills are "taken-forgranted, underlying, and usually unconscious assumptions" (Schein, 1990, p. 112) which comprise the core of the culture. However, Schein (1990) argue,

Deeply held assumptions often start out historically as values but, as they stand the test of time, gradually come to be taken for granted and then take on the character of assumptions. They are no longer questioned and they become less and less open to discussion (Schein, 1990, p. 112).

Moreover, this study shows that when values take the character of assumptions they disappear as explicit and practiced learning goals and transform into implicit expectations and this result highlights that implicit psychosocial skills are gradually less and less practiced in the environment. In the following, we will argue that this cultural characteristic of the club is a crucial problem for the young soccer player's possibility to learn psychosocial skills necessary for a successful transition from prospect to professional player in the club's first eleven.

In combination with the distinction between explicit and implicit psychosocial skills and as outlined previously in table 2, the study revealed four different combinations of psychosocial skills: 1) internal implicit skills, 2) internal explicit skills, 3) interpersonal implicit skills and 4) interpersonal explicit skills. Especially category one and three are of importance due to the implicit nature 
and the problems associated with psychosocial skills being "caught" instead of "taught" (Gould \& Carson, 2008). The lack of explication of these psychosocial skills additionally represents a problem, as the young soccer players may not be aware of the implicit psychosocial skills (taken-for-granted, underlying, and unconscious assumptions) they have developed, and therefore, these skills may be less usable for their career. Furthermore, the environment may not be aware of teaching strategies encouraging the development of these skills in players who are not displaying the skills from the very start (Martindale \& Mortimer, 2011).

An important characteristic in the case study were the participants' explicit focus on what they believe are the espoused values and thus important psychosocial skills to learn, especially "self awareness", "the ability to work hard", and "motivation". However, previous research (Van Yperen, 2009; Holt \& Dunn, 2004) show that implicit psychosocial skills such as "managing performance and process outcomes" and "goal setting" (two of the mainly implicit internal psychosocial skills) and "utilize team skills" and "general social skills" (two of the mainly implicit interpersonal psychosocial skills) may be even more important psychosocial skills for the players in negotiating the transition to professional soccer and meeting future coaches and team mates outside the familiar environment at AGF. This point is clarified by what the professional players in the case study experienced as the pivotal psychosocial skills in their transition to professional soccer, namely "managing performance and process outcomes" (i.e. the ability to learn new skills; learning to handle adversity; dealing with pressure; staying focused despite setbacks and setting own goals), which is consistent with previous research regarding the pathway to elite performance (MacNamara et al., 2010; MacNamara, 2011). However, a number of these psychosocial skills were not talked about or taught to the youth players in any explicit way, and therefore it seems coincidental that the players are aware of the importance of developing and displaying these skills. Despite the need for these psychosocial skills they were only indirectly practiced and rarely talked about in the environment and on academy level, and thus constitute a frailty and challenging task in the culture.

Finally, as a result, and taking into account the professional players experience, these psychosocial skills - or more precisely: the lack of these skills represent the almost unbridgeable "gap" (or glass ceiling) between academy level (youth soccer) and elite level (professional soccer), which is the most important transition in a young player's career (MacNamara et al., 2010; MacNamara, 2011; Van Yperen, 2009). Therefore it seems crucial that, coaches, environments and youth soccer academies are aware of the differences between discourse and practice of psychosocial skills on academy level, which psychosocial skills are relevant for success on academy level and which psychosocial skills are needed for career success and coping with transitions to professional soccer. 


\section{Conclusions}

The current study demonstrates the interweaving of adolescent athletes psychosocial skills and the talent development environment, and thus underlines the social construction of psychosocial skills in elite sport. This study shows that internal and interpersonal psychosocial skills are equally important for the soccer players in the case study. However, very few interpersonal psychosocial skills are explicit and practiced in the environment, whereas more internal psychosocial skills are practiced, incorporated into the culture and part of the shared articulated discourse in the environment. This result may inspire practitioners to work explicitly with psychosocial skills in relation to the sporting environment in which they are developed. Although this case study is unique, i.e. based on Danish culture and a specific sporting environment, the aim to explore and identify explicit and implicit psychosocial skills may provide inspiration for those involved in talent development environments across cultures and especially in teaching adolescents to cope with transitions. The distinction between and the awareness of explicit and implicit psychosocial skills in specific environments may provide a tool for optimizing talent development and soccer environments in Denmark and other countries.

\section{Endnotes:}

${ }^{1}$ For further information about ESAA visit: http://www.esaa.dk/content/english

${ }^{2}$ Arrangements for anonymity are accepted by the club and the participants.

\section{Acknowledgement}

We wish to express our gratitude to AGF and the participant in the case study who gave their time to take part in interview and informal talks. We also thank the Ministry of Culture Committee on Sports Research who allocated grants to the research project thereby made it possible to realize. The authors have no potential conflicts of interest 


\section{References}

Alfermann, D. \& Stambulova, N. (2007). Career transitions and career termination. In R. Eklund \& G. Tenenbaum (Eds.), Handbook of Sport Psychology (3 ed., pp. 712-736). New York: Wiley.

Brettschneider, W. D. (1999). Risks and Opportunities: Adolescents in Top-Level Sport - Growing Up with the Pressures of School and Training. European Physicial Education Review, 5, 121-133. doi: 10.1177/1356336X990052004

Bronfenbrenner, U. (1979). The ecology of human development. Cambridge: Harvard University Press.

Bronfenbrenner, U. (1999). Environments in developmental perspective: Theoretical and operational models. In S. L.Friedmann \& T. D. Wachs (Eds.), Measuring environments across life span: Emerging methods and concepts (pp. 3-28). Washington DC: American Psychological Association Press.

Chamberlain, G. P. (2006). Researching strategy formation process: An abductive methodology. Quality and quantity, 40(2), 289-301. doi: 10.1007/s11135-0058094-3

Christensen, M. K. \& Soerensen, J. K. (2009). Sport or school? Dreams and dilemmas for talented young Danish football players. European Physical Education Review, 15, 1-18. doi: 10.1177/1356336X09105214

Côté, J., Lidor, R., \& Hackfort, D. (2009). ISSP Position Stand: To Sample or to Specialize? Seven Postultes About Youth Sport Activities That Lead to Continued Participation and Elite Performance. International Journal of Sport and Exercise Psychology, 7, 7-17. doi: 10.1080/1612197X.2009.9671889

Danish, S. J., Petitpas, A. J., \& Hale, B. D. (1995). Psychological interventions: A life development model. In S. M. Murphy (Ed.), Sport psychology interventions (pp. 19-38). Champaign, IL: Human Kinetics.

Dubois A. \& Gadde, L.-E. (2002). Systematic combining: an abductive approach to case research. Journal of Business Research, 55, 553-560. doi:10.1016/S01482963(00)00195-8

Flyvbjerg, B. (2006). Five Misunderstandings About Case-Study Research. Qualitative Inquiry, 12, 219-245. doi:10.1177/1077800405284363 
Gould, D. \& Carson, S. (2008). Life skills development through sport: current status and future directions. International Review of Sport and Exercise Psychology, 1, 58-78. doi: 10.1080/17509840701834573

Harwood, C. G. (2008). Developmental Consulting in a Professional Football Academy: The 5Cs Coaching Efficacy Program. The Sport Psychologist, 22, 109133. Retrieved from http://journals.humankinetics.com/tsp

Helsen, W. F., Hodges, N. J., Winckel, J. V., \& Starkes, J. L. (2000). The roles of talent, physical precocity and practice in the development of soccer expertise. Journal of Sports Sciences, 18, 727-736. doi: 10.1080/02640410050120104

Henriksen, K., Stambulova, N., \& Roessler, K. K. (2010). Holistic approach to athletic talent development environments: A successful sailing milieu. Psychology of Sport and Exercise, 11, 212-222. doi:10.1016/j.psychsport.2009.10.005

Holt, N. L. \& Dunn, J. G. H. (2004). Toward a Grounded Theory of the Psychosocial Competencies and Environmental Conditions Associated with Soccer Success. Journal of Applied Sport Psychology, 16, 199-219. doi: 10.1080/10413200490437949

Janelle, C. \& Hillman, C. (2003). Expert performance in sport: current perspectives and critical issue. In J. L.Starkes \& K. A. Ericsson (Eds.), Expertperformance in sports (pp. 19-48). Champaign, IL: Human Kinetics.

Jones, M. I. \& Lavallee, D. (2009). Exploring the life skills needs of British adolescent athletes. Psychology of Sport and Exercise, 10, 159-167. doi:10.1016/j. psychsport.2008.06.005

Kruuse, E. (2008). Kvalitative forskningsmetoder [Qualitative Research Methods]. (6 ed.) Copenhagen: Dansk Psykologisk Forlag.

Kvale, S. \& Brinkmann, S. (2009). Interviews: Learning the Craft of Qualitative Research Interviewing (2 ed.) Thousand Oaks, California: Sage Publications.

Lincoln, Y. \& Guba, E. (1985). Naturalistic Research. Beverly Hills, CA: Sage Publications.

Maaloe, E. (2004). In Case of Case Research. Department of Organization and Management. 
MacNamara, A. (2011). Psychological characteristics of developing excellence. In D. Collins, H. Richards, \& A. Button (Eds.), Performance Psychology (pp. 4764). Kidlington: Elsevier.

MacNamara, A., Button, A., \& Collins, D. (2010). The Role of Psychological Characteristics in Facilitating the Pathway to Elite Performance. Part 1: Identifying Mental Skills and Behaviors. The Sport Psychologist, 24, 52-73. Retrieved from http://journals.humankinetics.com/tsp

Martindale, R. J. J. \& Mortimer, P. (2011). Talent Development Environments - Key Considerations for Effective Practice. In D. Collins, H. Richards, \& A. Button (Eds.), Performance Psychology (pp. 61-77). Kidlington: Elsevier.

Olesen, S. G. (2001). Om observation i institution og skole [About observation in institutions and schools]. Viborg: Forlaget PUC.

Patton, M. Q. (2002). Qualitative evaluation and research methods. Newsbury Pard, CA: Sage Publications.

Ramian, K. (2007). Casestudiet i praksis [The Case Study in praxis]. (1 ed.) Aarhus: Academica.

Robson, C. (2002). Real World Research. Oxford: Blackwell.

Schein, E. H. (1990). Organizational Culture. American Psychologist, 45, 109-119. doi:10.1037/0003-066X.45.2.109

Spradley, J. P. (1980). Participant observation. Fort Worth: Harcourt Brace College Publishers.

Van Yperen, N. W. (2009). Why Some Make It and Others Do Not: Identifying Psychological Factors That Predict Career Success in Professional Adult Soccer. The Sport Psychologist, 23, 317-329. Retrieved from http://journals. humankinetics.com/tsp

Yin, R. K. (2009). Case Study Research: Design and Methods. (4 ed.) Thousands Oaks, California: Sage Publications. 
Carsten Hvid LARSEN is a Ph.D. Fellow at the University of Southern Denmark, Institute of Sports Science and Clinical Biomechanics and a sport psychologist at the professional soccer team - Odense Soccer Club. He is the current President of the Danish Sport Psychology Association (2010-). E-mail address: chlarsen@health.sdu.dk

Dorothee ALFERMANN is a Professor, Ph.D., at the University of Leipzig, Institute of Sport Psychology and Sport Pedagogy. She is the former Co-Editor in Chief at Psychology of Sport \& Exercise and currently the President of the German Sport Science Association. She is author of several publications within her field of interest. E-mail address: alfermann@uni-leipzig.de

Mette Krogh CHRISTENSEN is an Associate Professor, Ph.D., at the University of Southern Denmark, Institute of Sports Science and Clinical Biomechanics. She is Chief of the Research Group of Talent Development and Expertise and has published several articles in his field of interest. E-mail address: mkchristensen@health.sdu.dk 